\title{
Comunicación estratégica y el uso de las redes sociales para la supervivencia de las instituciones culturales con la COVID-19
}

Patricia Alonso Arroba | artista visual y diseñadora gráfica

URL de la contribución <www.iaph.es/revistaph/index.php/revistaph/article/view/4797>

Desde la aparición de Internet y, especialmente, tras la explosión a nivel mundial del empleo de las redes sociales en los últimos años, las instituciones culturales han observado que debían actualizarse continuamente para así poder comunicar con el público. El uso de nuevas tecnologías tanto en el diseño de exposiciones como en la red ha permitido transmitir la cultura de una manera más cercana, amena y atractiva a los futuros visitantes reavivando su interés por el sector y contribuyendo así a su mantenimiento también a nivel económico.

En este contexto las instituciones dedicadas a la cultura han conseguido actualizarse poco a poco en la

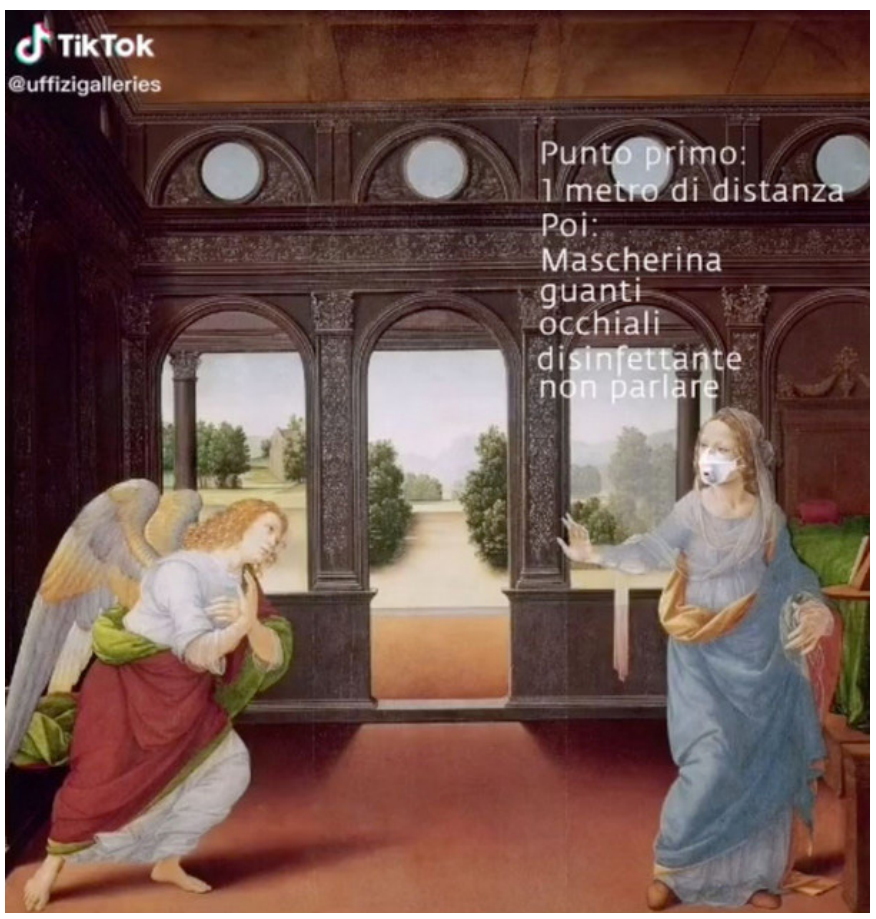

Imagen de Tik Tok promocional de la Galeria Uffizi de Florencia última década, incluyendo en sus planes de comunicación estrategias como, por ejemplo, las visitas virtuales a sus instalaciones o reavivando el interés de sus usuarios interactuando con ellos a través de las redes sociales, dedicando su tiempo a escuchar sus comentarios y sugerencias. Se trataba de un recurso más de comunicación pero no el pilar principal de los objetivos principales de dichas instituciones, sino un medio para atraer a futuros usuarios a visitar sus instalaciones, publicitarlas y otorgarles difusión.

No obstante, este escenario cambia a partir de marzo de 2020, en España, y dos semanas antes, en Italia, cuando ambos países declaran el estado de alarma ante la crisis sanitaria de la COVID-19 y toda la población permanece confinada. Las repercusiones no se hacen esperar y en las redes sociales aparecen multitud de alternativas como las visitas a los museos virtuales, los espectáculos online, el acceso gratuito a libros y documentales y un largo etcétera. Es el principio de una latente revolución de las instituciones culturales que ven peligrar ya su bastante frágil situación en los últimos años.

Según el Consejo Internacional de museos (ICOM), el $95 \%$ de los cerca de 60.000 museos existentes en el mundo permanecen cerrados a causa de la pandemia (ICOM 2020) y si la situación se prorroga durante un gran tiempo una importante cantidad de ellos se verán avocados al cierre. Solo sobrevivirán aquellos que debido a la accesibilidad de infraestructuras y obtención de nuevas tecnologías se adapten mejor a las necesidades para los usuarios de manera virtual. Ejemplos de ello los recoge la revista Life and Style (2020), como es el caso del Museo del Louvre que, desde su cierre y gracias al creciente empleo de herramientas digitales, ha visto multiplicadas por cuatro las conexiones a su 
a debate Comunicación y redes sociales en instituciones culturales

| coordina Candela González Sánchez

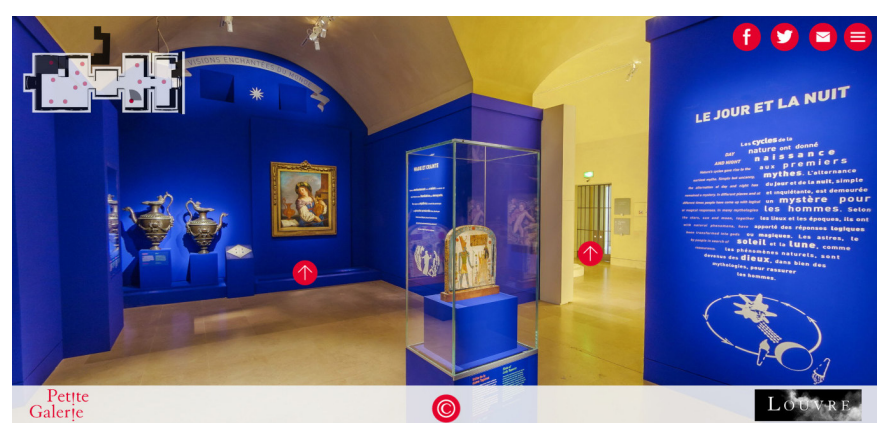

Visita virtual a la exposicion Founding Myths from Hercules to Darth Vader. Petite Galerie del Museo del Louvre

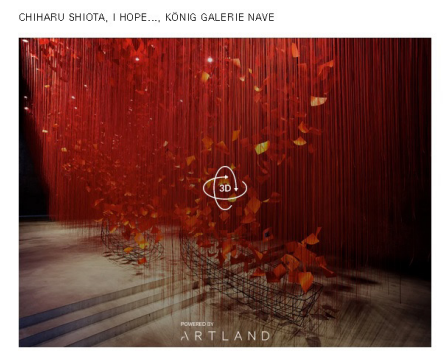

CLAUDIA COMTE, JNMGLE AND CORALS. MONNIG GALERIE CHAFEL
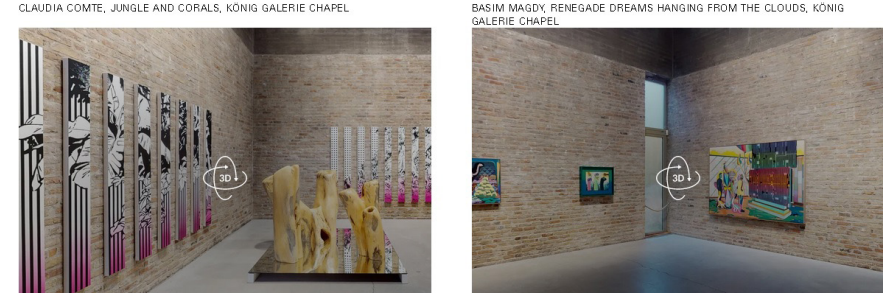

Algunas de las visitas virtuales propuestas por la Galería Koenig

página web, hasta alcanzar 400.000 visitas al día, o la galería Koenig cuyo propietario, Johann Koenig, a través de Instagram, realiza en directo entrevistas a diferentes artistas sobre su trabajo. Otro caso de adaptación y uso de las nuevas tecnologías que más repercusión ha tenido ha sido la apuesta de la Galleria Uffizi con su inversión en las redes sociales; primero en Facebook, donde ya posee más de 18 millones de seguidores y, posteriormente, con TikTok, una plataforma que, según el director del museo, Eike Schmidt, permite alcanzar un target más joven a través de la creación de breves videos de hasta 15 segundos que explican con un carácter divertido e ironía algunas de las grandes obras de arte del museo (Giraud 2020).
Recientemente, el MiBACT (Ministero per i beni e le attività culturali e per il turismo) publicó un documento (2020), enviado por el Politecnico de Milano, celebrando un notable incremento de la interacción de los usuarios a través de las redes sociales con los museos italianos durante el periodo de confinamiento durante la pandemia (Monti 2020). Esta noticia debería ser positiva ya que ha estimulado a las organizaciones culturales a crear nuevas modalidades online de interacción con el público. No obstante, dicho triunfalismo no debería ser tal ya que no se sabe aún con certeza si esta interacción permanecerá en el futuro cuando no exista una pandemia y la sociedad vuelva a la "nueva normalidad".

Otro factor positivo en relación con el incremento de las visitas a las instituciones culturales online y a través de las redes sociales es que nos indica que el público necesita humana y psicológicamente una oferta cultural cada vez más vasta y creativa. Sin duda, el periodo de confinamiento ha contribuido a ello y estos medios tecnológicos han sido fundamentales a la hora de satisfacer esas inquietudes culturales; no obstante, deberíamos preguntarnos a qué precio.

Si por una parte las dificultades que han tenido y aún arrastran las instituciones culturales en algunas ocasiones han provocado una emergencia de la creatividad e reinvención de estrategias para llegar al público, en otras se podría decir que se han convertido en productos de consumo listos para devorar por unos usuarios hambrientos de la misma durante el periodo de pandemia. Sería interesante analizar cuáles son los límites del uso de las herramientas tecnológicas y las redes sociales para no banalizar un sector tan precioso y valioso para la humanidad como es nuestro patrimonio cultural.

Los objetivos que a largo plazo las instituciones culturales deberían supervisar, y si la economía lo permite, son estar constantemente actualizadas a niveles de tecnología, comunicación y nuevos instrumentos que la sociedad propone, pero siempre teniendo en cuenta unos factores donde lo fundamental es la comunicación de la cultura y la transmisión de sus valores. 


\section{BIBLIOGRAFÍA}

- Giraud, C. (2020) Gli Uffizi sbarcano su Tik Tok. Umorismo al museo con clip scanzonate di grandi capolavori. Artribune, 29 de abril de 2020. Disponible: https://www.artribune.com/artivisive/2020/04/uffizi-su-tiktok/ [Consulta: 11/12/2020]

- ICOM (2020) Boletín semanal Cultura \& Covid-19, Impacto \& Respuesta, n. ${ }^{\circ} 4$. Disponible en: https://es.unesco.org/ sites/default/files/issue_4_es_culture_covid-19_tracker-2.pdf [Consulta: 11/12/2020]

- Los recorridos virtuales de los museos más visitados del mundo. Life \& Style, 9 de abril de 2020. Disponible en: https://lifeandstyle.expansion.mx/entretenimiento/2020/04/09/ recorridos-virtuales-de-los-museos-mas-visitados-del-mundo [Consulta: 11/12/2020]

- Ministero per i Beni e le Attività Culturali (2020) La reputazione online di musei, parchi archeologici, istituti e luoghi della cultura italiani-Report relativo al mese di maggio 2020. Disponible en: http://musei.beniculturali.it/wp-content/uploads/2020/07/ Report-Reputazione-Online-dei-Musei_Maggio-2020_sintesi. pdf [Consulta: 14/12/2020]

- Monti, S. (2020) Musei e social media in pandemia: opportunità mancata? Artribune, 22 de julio de 2020. Disponible en: https://www.artribune.com/progettazione/ new-media/2020/07/musei-social-media-pandemia-analisi/ [Consulta: 14/12/2020] 